\title{
¿PUEDE LA NUEVA GESTIÓN PÚBLICA FORTALECER LA TRANSPARENCIA GUBERNAMENTAL?: EL CASO DE LA GESTIÓN DEL DESEMPEÑO
}

\author{
Ernesto Velasco Sánchez \\ Cívicus, Consultores en Gestión Pública y Social, México \\ ernestovelascos@gmail.com
}

\begin{abstract}
RESUMEN
El artículo indaga sobre si las reformas estilo Nueva Gestión Pública (NGP) y aquellas orientadas a fomentar la transparencia en el sector público son compatibles, es decir, si se refuerzan mutuamente o son contradictorias. Para ello se propone analizar las sinergias y las tensiones que existen entre la transparencia y una de las herramientas más utilizadas dentro del arsenal de la NGP: la gestión del desempeño. Para ello se presentan diferentes formas entender la idea de transparencia y se identifican distintas estrategias adoptadas por la gestión del desempeño. A continuación se identifican sinergias y potenciales contradicciones entre gestión por resultados y transparencia. Se concluye que la relación entre estos conceptos es altamente contextual, es decir, depende del tipo de organización del que se trate, de las actitudes que adopten los actores involucrados y de cuándo y cómo se publique la información sobre el desempeño. De allí que se enfatice la necesidad de diseñar tanto los regímenes de transparencia y como de gestión de desempeño que respondan de manera adecuada a contextos y necesidades específicos.
\end{abstract}

Palabras clave: Transparencia, Gestión del desempeño, Gestión pública, Reforma administrativa. 


\title{
CAN THE NEW PUBLIC MANAGEMENT STRENGTHEN GOVERNEMENTAL TRANSPARENCY?: THE CASE OF PERFORMANCE MANAGEMENT
}

\begin{abstract}
The article explores whether the new public management style reforms (NPM) and those aimed to promoting transparency in the public sector are compatible, i.e., if they reinforce each other, or if they are contradictory. In order to answer this question, the article proposes to analyze the synergies and tensions which exist between transparency and one of the most frequently used tools in the arsenal of the NPM: performance management. In order to accomplish this, different ways of understanding the concept of transparency are presented and various strategies adopted by performance management are identified. Subsequently, synergies and potential contradictions between the management of performance and transparency are identified. The main conclusion is that the relationship between these two concepts is highly contextual, i.e., it depends on the type of organization, the attitudes adopted by those involved, and when and how information about performance management is published. Consequently, the need of designing both transparency and performance management regimes, to appropriately respond to specific contexts and requirements, is emphasized.
\end{abstract}

Keywords: Transparency, Performance management, Public management, Administrative reform. 


\section{INTRODUCCIÓN}

En la actualidad existe un amplio consenso sobre el valor de la transparencia como un componente fundamental de la buena gobernanza de las naciones ${ }^{1}$. En buena medida, la capacidad social de control del gobierno depende de que la ciudadanía sea capaz de conocer cómo actúa el gobierno, de manera que pueda hacerse un juicio sobre su funcionamiento y asignar reconocimientos y sanciones en caso de actuaciones sobresalientes o deficientes, respectivamente. Así, no se puede considerar que el gobierno entrega cuentas si no existe acceso suficiente y oportuno a la información requerida para valorar las actividades gubernamentales (Steward 1984). Por lo tanto, la transparencia es un elemento indispensable que diferencia a las democracias de las tiranías electas (Day y Klein 1987).

Este asunto resulta particularmente importante dado que en el Estado contemporáneo es frecuente que las decisiones que mayor efecto tienen sobre la vida de las personas son tomadas por funcionarios públicos que laboran en las oficinas gubernamentales, lejos de los reflectores y la atención de los medios y del ciudadano común (Aucoin y Heintzman 2000). La complejidad del sector público actual exige cada vez mayores niveles de delegación de ámbitos de decisión y acción hacia estructuras administrativas centralizadas o hacia organizaciones especializadas, lo que genera problemas de control y coordinación importantes, así como un alto riesgo de insensibilidad frente a las necesidades y demandas de la ciudadanía. En este sentido, el crecimiento de las burocracias y de los cuerpos profesionales conlleva el riesgo de crecientes espacios de opacidad.

Ahora bien, en las últimas tres décadas se han desarrollado movimientos de reforma administrativa que, si bien no todos han tenido a la transparencia como un fin último, sí han tratado de recuperar el sentido público de la acción gubernamental que se había perdido de vista justamente por el crecimiento y la introversión del sector público (Aguilar 2006). Una primera ola de cambios se orientó a redimensionar el tamańo del sector público, lo que implicó la desaparición de organismos y programas gubernamentales, la privatización de activos públicos, el recorte o control del crecimiento de los presupuestos, y el despido de personal. Sin embargo, este modelo redimensionador encontró rápidamente sus límites, toda vez que aún se exigían soluciones públicas a diversos problemas, y por la imposibilidad de trasladar al sector privado muchas actividades cuyas características impedían su adecuada provisión por medio del mercado. Una segunda ola de reformas, que tuvo lugar particularmente desde mediados de la década de 1980, ha incorporado los argumentos críticos frente a la

1 Véase, por ejemplo, Kondo (2002). 
administración pública tradicional, pero considera que la introducción de herramientas adecuadas de gestión puede hacer a las organizaciones públicas igual e incluso superiores en su desempeño en comparación con sus contrapartes empresariales. A este conjunto de reformas se le ha denominado como Nueva Gestión Pública (NGP), entendida como un movimiento de reforma administrativa que promueve la introducción en el sector público de presiones competitivas (marquetización) y de enfoques gerenciales desarrollados en el sector privado (gerencialismo), para lograr mayor eficiencia, eficacia y recuperar la confianza de los ciudadanos en la acción del gobierno (Hood 1991). Posteriormente, durante la década de 1990 y la primera del siglo XXI, un creciente número de países han introducido legislación para asegurar el acceso a la información pública gubernamental: mientras en 1990 sólo trece países contaban con leyes en la materia, para el 2011 ya eran ochenta y una veintena más trabajaban en su aprobación (Wignaraja y Gebrehiwot 2011). Este tipo de reformas se concentra más en los aspectos de legitimidad del ejercicio del poder, por medio de acercar o abrir el gobierno a los ciudadanos, más que aumentar la capacidad administrativa del gobierno.

A partir de lo anterior se puede ver que, si bien en algunos países la NGP y las reformas en pro de la transparencia gubernamental son contemporáneas, se orientan a resolver problemas o retos diferentes de la reforma administrativa. El presente texto hace énfasis en establecer las dimensiones conceptuales de la idea de transparencia, así como de la que se refiere a la gestión del desempeño. Su intención es entender cómo estas dos variables pueden relacionarse en diferentes escenarios o momentos e la acción pública, a partir de retomar tanto discusiones de carácter teórico como aquellos de corte empírico. La pregunta que se plantea este ensayo es si estas dos corrientes de cambio en el gobierno son compatibles, es decir, si se refuerzan mutuamente, o si son contradictorias. Para aproximar una respuesta, se propone analizar las sinergias y las tensiones que existen entre la transparencia y una de las herramientas más utilizadas dentro del arsenal de la NGP: la gestión del desempeño. En la primera sección se ofrecen los conceptos que se utilizarán a lo largo del texto. En la segunda se analizan las sinergias potenciales entre la gestión del desempeńo y la transparencia, mientras que en la tercera se señalan las contradicciones que podrían existir entre ambas. En la última parte se ofrecen algunas reflexiones a modo de conclusión. 


\section{CONCEPTOS DE TRANSPARENCIA Y DE GESTIÓN DEL DESEMPEÑO}

La idea de transparencia es una metáfora más que un concepto acabado, es decir, un símbolo que naturalmente es ambiguo y que, por lo tanto, puede ser interpretado de muy diferentes formas, algunas incluso contradictorias. En general, la idea detrás de la transparencia debe mucho a la tradición liberal del pensamiento político que señala la necesidad de evitar el abuso de poder por parte de los gobernantes. La salida que se ha propuesto es asegurar las condiciones para que no sólo exista equilibrio de poderes dentro del Estado, sino que permitan la participación social activa que convierta a cada ciudadano en una fuente potencial de vigilancia y control del sector público (Guerrero 2005, Vergara 2008). Christopher Hood (2006) identifica cuatro formas de entender la transparencia que tienen un recorrido histórico de larga data:

1. Transparencia como gobierno de reglas. Es una idea que aparece de manera recurrente desde la antigüedad en Oriente y Occidente es que los gobiernos deben basar su actividad en normas y regulaciones estables y conocidas, es decir, no arbitrarias, en contraposición a confiar el destino de la comunidad política únicamente a las capacidades, destrezas y carácter moral del gobernante. La idea de Estado de Derecho se relaciona directamente con la idea de transparencia así interpretada.

2. Trasparencia como candor y franqueza. Se refiere a la exigencia de que los asuntos gubernamentales y de interés de la sociedad se discutan y manejen a la vista de todos y con verdad.

3. Transparencia como libre acceso a la información pública. Instituida por primera vez en la ley sueca de libertad de prensa de 1766 que incluía el derecho a acceder a los expedientes gubernamentales, ésta es una forma frecuente de entender en nuestros días la expresión transparencia, particularmente derivada de la aprobación de la Ley de Libertad de Información de los Estados Unidos de 1966 y de la llamada Ley Sunshine de 1976, que han influido el marco legal de muchos países como el de México. Para algunos autores ésta es la forma más precisa y práctica de entender la transparencia (Guerrero 2005).

4. Transparencia como transparencia social. El uso de la ciencia para iluminar aquellos espacios o asuntos sociales donde ocurren injusticias o desdichas. Se trata de la posibilidad de hacer visibles y debatir abiertamente aquellas situaciones sociales que normalmente pasan 
desapercibidas o que parecen no se cuestionan debidamente, para problematizarlas y proceder a su atención.

Adicionalmente, Hood (2006) identifica otros significados de transparencia que han aparecido desde el siglo XX, entre los que destacan los siguientes:

1. Transparencia como participación ciudadana en la toma de decisiones. Encarnada en mecanismos como las sesiones deliberativas abiertas, esta idea de transparencia entiende que la toma de decisiones colectiva debe partir del intercambio de argumentos entre gobierno y sociedad.

2. Transparencia como regímenes claros de contabilidad. Idea particularmente impulsada desde los ańos 1990 por organizaciones financieras multilaterales, se refiere a contar con criterios o estándares contables que permiten conocer cómo gasta sus recursos el gobierno, de manera que sea posible conocer el costo de las diferentes actividades y revelar posibles subsidios cruzados entre ellas.

El recuento anterior permite ver que la idea de la transparencia es multidimensional y que se requiere tener cuidado en no dar por sentado que tiene un sentido inequívoco. Por otra parte, respecto de la Nueva Gestión Pública, un elemento central de las doctrinas gerencialistas se refiere a recuperar el principio de que las organizaciones públicas existen para satisfacer demandas y necesidades ciudadanas, por lo cual se debe poner al ciudadano en el centro de la atención, de manera que se le otorgue la debida consideración a sus puntos de vista en el diseño, la prestación y la evaluación de los bienes y servicios proveídos por el gobierno. La gestión de desempeño forma parte de este esfuerzo por cerrar la brecha entre los resultados esperados y aquellos que efectivamente se alcanzan por parte de las organizaciones públicas. No existe una definición única y aceptada de qué es la gestión del desempeño (o del rendimiento), aunque en general se trata de un conjunto de herramientas que siguen el siguiente principio básico:

$[U]$ na organización pública determina su desempeño deseado y señala la forma en que dicho rendimiento será medido por medio de la definición de indicadores de desempeño. Una vez que la organización ha realizado sus actividades, será posible demostrar si el desempeño proyectado fue alcanzado y a qué costos (De Brujin 2001: 7) $)^{2}$.

2 El principio básico de la gestión del desempeño no es nuevo. De hecho, ha sido el resultado de una evolución en las prácticas de planificación y programación de todo el mundo. El primer esfuerzo claro para medir el rendimiento se puede encontrar desde la década de 1960 en la experiencia de Estados Unidos y Suecia en la gestión 
Como se puede observar, el corazón de la gestión del desempeño es la definición de los indicadores ${ }^{3}$.

Desde la década de 1980, y con gran fuerza en la actualidad ${ }^{4}$, se ha tratado de traducir estos grandes principios en herramientas que permitan medir y tomar decisiones respecto del rendimiento gubernamental, al grado de que hay autores que señalan que las reformas administrativas contemporáneas reflejan una obsesión con el desempeño (Radin 2006, Talbot 2010). De inmediato surge la pregunta de a qué se debe esta proliferación de esquemas de medición del rendimiento gubernamental. La respuesta, de acuerdo a la teoría administrativa, se encuentra en que la ambigüedad de los objetivos es uno de los elementos a los que se atribuye la falta de eficacia tanto en organizaciones públicas como privadas 5 . En otras palabras, la falta de claridad sobre lo que se desea de la organización y de sus miembros genera una disminución de su capacidad para producir los bienes, servicios, regulaciones, entre otros, que debe producir. Los principales beneficios de este enfoque, de acuerdo a la literatura, son los siguientes: 1) se aclara la relación entre las aportaciones y resultados; 2) permite medir el progreso hacia los objetivos del gobierno e incluso recompensar o sancionar el desempeño individual y de la organización; y 3) facilita la asignación de responsabilidades por el rendimiento (De Brujin 2001: 8). Además, la

por objetivos y en los contratos de desempeño en Francia, y durante la década de 1970 en los presupuestos de base cero. Lo novedoso de la última ola de medición del desempeño quizás se encuentra en el énfasis en vincular los insumos no sólo a los resultados inmediatos de la acción sino a los impactos sociales que se producen. Durante la década de 1980, el giro hacia los productos y los resultados estaba definitivamente arraigado en los Estados Unidos, Nueva Zelanda, el Reino Unido y Australia (Heinrich 2002).

3 Del latín indicare, significa: "mostrar o significar algo, con indicios y señales". Nuevamente, no existe consenso sobre qué constituye un indicador. El mundo de la práctica resulta más ilustrativo que la literatura. De acuerdo con el gobierno chileno, un indicador es "una herramienta que entrega información cuantitativa respecto del logro o resultado en la entrega de productos (bienes o servicios) generados por la institución, que cubre aspectos cuantitativos o cualitativos" (DIPRES 2003).

4 Es posible encontrar ejemplos de esto en países desarrollados y en vías de desarrollo, entre los que destacan: el intensivo uso de indicadores de rendimiento en los ámbitos nacional y local y la Ley de Resultados y Desempeńo Gubernamental de 1997 en el Reino Unido, la Agenda Presidencial de Gestión del 2001 y la Herramienta de Valoración de Programas de 2002 en los Estados Unidos, el Programa de Mejoramiento de la Gestión en Chile, el Programa Resultados para Canadá y las auditorías de desempeńo en Canadá y los presupuestos basados en desempeńo en los Países Bajos.

5 De hecho, en organizaciones gubernamentales, un estudio reciente muestra una relación inversa entre ambigüedad de objetivos (la amplitud para traducirlos en guías operativas, indicadores y prioridades) y la simplificación de los trámites y los procedimientos internos (red tape), la descentralización administrativa de las decisiones dentro de las instituciones, las expectativas que tienen los funcionarios de ser adecuadamente recompensados por su esfuerzo y con la satisfacción laboral (Chun y Rainey 2006). 
gestión del rendimiento puede promover un proceso de mejora continua de los servicios públicos mediante la extracción de lecciones de buenas prácticas y la identificación de oportunidades para el cambio (Comisión de Auditoría 2000: 6). Colin Talbot (2010) ha identificado cuatro tipos de estrategias o grupos de herramientas que tienen como propósito mejorar el desempeño de las organizaciones. A saber:

1. Establecer relaciones contractuales con los gerentes. Se trata de establecer formalmente compromisos de mejora y objetivos de desempeño a alcanzar, o el establecimiento y revisión del apego a estándares mínimos de calidad por parte de los prestadores de servicios públicos.

2. Intervenciones orientadas a generar capacidades. En general, se refieren a acciones que tienen por objetivo mejorar las actividades relacionadas con el desempeńo, es decir, no se trata de definir objetivos o estándares, sino mejorar directamente las operaciones y los comportamientos que determinan el rendimiento organizativo.

3. Introducción de mecanismos de mercado o similares al mercado. Se orientan a promover la competencia dentro del sector público, de manera que se mejore la eficiencia y la capacidad de innovación al reducir el poder monopólico de las organizaciones burocráticas.

4. Introducción de mecanismos para dar voz y oportunidades de elección al usuario. Se trata de intervenciones que reviertan las asimetrías de información entre los ciudadanos y los operadores de los programas y servicios, de manera que los usuarios conozcan mejor sus derechos y puedan incluso elegir a su proveedor de servicios públicos.

En las secciones siguientes se exploran las relaciones entre transparencia y estas herramientas de gestión del desempeño, señalando primero las potenciales sinergias o complementariedades entre ellas, para luego identificar sus posibles contradicciones.

\section{LAS SINERGIAS ENTRE TRANSPARENCIA Y GESTIÓN DEL DESEMPEÑO}

¿Existen sinergias entre las herramientas de la gestión del desempeño y la transparencia gubernamental? Frente a esta pregunta, la literatura normalmente establece una relación directa y positiva entre ambos conceptos al señalar que la transparencia genera los incentivos adecuados para que el comportamiento de las organizaciones se apegue lo más posible a las expectativas sociales (Arellano 2005, Vergara 2008). Es decir, 
transparencia y gestión del desempeño se alimentan mutuamente, ya que las organizaciones que declaran abiertamente sus propósitos, permiten un mejor escrutinio público que, a su vez, genera incentivos más fuertes para lograr un rendimiento superior. La transparencia abona también al desempeño de otra forma, en la medida en que facilita la coordinación dentro y entre las organizaciones públicas, de manera que la información fluya con mayor facilidad entre los funcionarios que la requieren para hacer su trabajo (Vergara 2008), y permite integrar mejor los esfuerzos en el caso de políticas transversales de manera que los resultados se valoren en conjunto y no fraccionados por las fronteras jurisdiccionales de cada organización gubernamental en lo individual (James 2005).

Ahora bien, este argumento general puede ser analizado con mayor profundidad si se vincula la aportación que hacen las diferentes estrategias de gestión del desempeño a las diferentes formas de entender la transparencia. Con respecto al primer significado de la transparencia identificado por Hood (2006), es decir, aquel que la entiende como el gobierno de reglas no discrecionales, resulta particularmente relevante la estrategia de establecer relaciones contractuales entre los directivos y los operadores de los servicios, ya que esto genera un marco de actuación dentro del cual se determina formalmente lo que se desea alcanzar y es posible, por lo tanto, valorar el desempeño organizativo según criterios acordados previamente. En este sentido, la gestión del desempeño puede reducir la arbitrariedad en la gestión pública.

Con respecto a la definición de transparencia como discusión franca y abierta de los asuntos públicos, las herramientas de la gestión del desempeño que pueden contribuir a una mayor transparencia son el establecimiento de relaciones contractuales (que permiten hacer públicos los compromisos de desempeño asumidos), la generación de capacidades (ya que pueden fortalecer las habilidades de los propios funcionarios para argumentar sus decisiones y demostrar su rendimiento), y la introducción de mecanismos de mercado (al permitir la comparación y la competencia abierta entre proveedores de bienes y servicios públicos).

La transparencia entendida como libre acceso a la información se ve apoyada por los mecanismos de gestión del desempeño en tanto promueven un mayor flujo de información entre gobierno y sociedad. Particularmente importantes en este caso son el establecimiento de relaciones contractuales y los mecanismos para dar voz y participación a los ciudadanos, ya que ambos permiten reducir las asimetrías de información entre el público y el aparato gubernamental. Por su parte, en lo que se refiere a entender la transparencia como visibilizar los problemas sociales, ésta se relaciona con la difusión de información sobre las causas de diversos problemas sociales 
y el seguimiento del comportamiento de los mismos a lo largo del tiempo. Relevante es en este caso la introducción de mecanismos de voz que permitan señalar dónde la gestión pública puede mejorar en la atención a dichas situaciones problemáticas que enfrenta la comunidad.

La transparencia entendida como la posibilidad de que los ciudadanos participen en la toma de decisiones puede promoverse por medio de la introducción de mecanismos de provisión competitiva de servicios, como es la publicación de tablas comparativas de desempeño (league tables), y dar voz y mecanismos de elección a los propios usuarios de los servicios. Finalmente, la transparencia vista como el establecimiento de regímenes claros de contabilidad gubernamental se relaciona con la gestión orientada a resultados de programas y servicios, particularmente mediante mecanismos como el establecimiento de presupuestos basados en resultados.

La discusión presentada se ha centrado en mostrar que, efectivamente, existen puntos de contacto importantes entre diferentes formas de entender la transparencia y las herramientas de la gestión del desempeño. En los casos señalados, es posible identificar una relación de reforzamiento mutuo: ciertas herramientas promueven cierto tipo de transparencia y esto, a su vez, puede ser un incentivo a un mejor rendimiento gubernamental. En consecuencia, se logra un mayor apego a uno de los valores básicos del buen gobierno democrático, al tiempo que se mejora el funcionamiento de las organizaciones públicas, elevándose simultáneamente, para usar los términos de Aguilar Villanueva (2011), la calidad institucional del gobierno y su calidad gerencial.

A pesar de esto, no en todas las situaciones esta relación entre transparencia y gestión del desempeño es positiva. En la sección siguiente se analizarán las situaciones en que la transparencia y la gestión del desempeño, más que reforzarse una a la otra, siguen lógicas contradictorias.

\section{TENSIONES ENTRE TRANSPARENCIA Y GESTIÓN DEL DESEMPEÑO}

Un creciente número de autores ha señalado que el contar con mayor transparencia no necesariamente genera mejor información ni tampoco mejor desempeño. Por ejemplo, Mary Graham advierte que los mecanismos de transparencia deben ser diseñados cuidadosamente o, de lo contrario, terminarán por generar grandes cantidades de información inútil (citada en Guerrero 2005: 30). Otros argumentan que el problema es que la transparencia implica la erosión de la confianza en la actuación de los propios funcionarios y profesionales expertos (como son médicos, trabajadores sociales, ingenieros, etc.), por lo que la introducción de 
mediciones y formas de seguimiento y monitoreo permanentes pueden llevar a un menor rendimiento organizativo y a la disminución de la calidad de los servicios (Arellano 2005, Radin 2006). También genera preocupación el que las demandas de transparencia generan cargas de trabajo y costos adicionales para la administración pública, lo que reduce su eficiencia y eficacia (Vergara 2008). Se ha señalado igualmente que hay actividades que para lograr sus objetivos deben mantenerse en secrecía, como es el caso de aquellas relacionadas con la seguridad nacional o con la verificación de la calidad de los servicios por medio de inspecciones sorpresa o el uso de usuarios simulados (Held 2006, Guerrero 2005). Finalmente, un tema recurrente se refiere a que puede aparecer una ilusión de transparencia, es decir, donde se tenga un mayor acceso información que no necesariamente es adecuada para conocer lo que verdaderamente ocurre dentro del gobierno: aparece el fenómeno que Fox denomina la transparencia opaca (Fox 2007). Esto hace evidente tanto la necesidad de usuarios capacitados para sacar ventaja de la información que se hace pública como también las posibles consecuencias negativas de que se produzca un desbordamiento de datos que impida un uso efectivo de los mismos (Heald 2006).

En esta sección se exploran cuatro asuntos en los que la transparencia y la gestión del desempeño se vuelven contradictorias, a saber: 1) cuando existen complicaciones para medir el desempeño; 2) cuando la transparencia puede generar comportamientos negativos en los funcionarios; 3) cuando la transparencia entra en tensión con la profesionalización de la función pública; y 4) cuando la transparencia de la información ocurre en un momento que puede afectar el rendimiento. Se finalizará con una discusión sobre de los efectos que tienen estas tensiones sobre el modelo de gestión del desempeño que se elija implantar en un momento dado. La premisa de la cual se parte es que tanto la transparencia como la gestión del desempeño visibilizan algunos fenómenos pero ocultan otros. Es decir, la decisión sobre qué se mide y qué se hace público implica también una decisión intencional o un resultado no intencionado respecto a lo que se va a mantener en la obscuridad.

\section{TranSPARENCIA Y COMPLICACIONES PARA MEDIR EL DESEMPEÑo}

La idea de que el acceso a la información permite a los ciudadanos valorar más adecuadamente las labores del gobierno requiere que dicha información sea clara y precisa. Sin embargo, en muchas ocasiones los funcionarios alegan que sus actividades no pueden ser medidas $y$, por lo tanto, que la difusión de datos que reflejan mal sus actividades puede llevar a apreciaciones incorrectas respecto al esfuerzo realizado. En otras palabras, el problema en este caso es que la transparencia puede llevar a 
emitir valoraciones que son inadecuadas o injustas respecto del desempeño organizativo.

Para analizar este problema, la literatura suele recurrir a las tipologías para determinar el grado de dificultad que tiene el medir su rendimiento. Una de las más citadas es la que ofrece James Q. Wilson en su libro Burocracia. Lo que las Agencias del Gobierno Hacen y Por Qué lo Hacen (1989), que propone diferenciar a las organizaciones de acuerdo a qué tan difícil es apreciar y medir los productos inmediatos de su actividad, así como la dificultad para medir los impactos o cambios en la realidad social que dichos productos generan. Al combinar estas dos dimensiones, se obtienen cuatro cuadrantes como se muestra a continuación.

\section{Tabla 1: La tipología de Wilson y la transparencia}

\section{Mensurabilidad de los productos}

\begin{tabular}{|c|c|c|}
\hline & Alta & Baja \\
\hline Alta & $\begin{array}{l}\text { Producción } \\
\text { (correos, recaudación de } \\
\text { impuestos) } \\
\text { La transparencia permite } \\
\text { informar adecuadamente } \\
\text { los juicios }\end{array}$ & $\begin{array}{l}\text { Artesanal } \\
\text { (servicios de salud, policía) } \\
\text { La transparencia no resulta } \\
\text { en una mejor comprensión } \\
\text { por parte del público de las } \\
\text { actividades realizadas }\end{array}$ \\
\hline Baja & $\begin{array}{l}\text { Procedimentales } \\
\text { (servicios de consejería, } \\
\text { atención a jóvenes delin- } \\
\text { cuentes) } \\
\text { La transparencia es posi- } \\
\text { ble en los procesos, pero } \\
\text { difícil en términos de los } \\
\text { impactos }\end{array}$ & $\begin{array}{l}\text { Organizaciones que "se las } \\
\text { arreglan" } \\
\text { (diplomacia, inteligencia) } \\
\text { Organizaciones opacas por } \\
\text { necesidad y por imposibili- } \\
\text { dad de ofrecer información } \\
\text { de desempeńo }\end{array}$ \\
\hline
\end{tabular}

Fuente: Elaboración propia a partir de Van Dooren, Bouckaert y Halligan (2010), que se basan en Wilson (1989). 
Wilson identifica cuatro tipos de agencias gubernamentales. En primer lugar, las organizaciones de producción, que son aquellas en que tanto los productos inmediatos de su actividad (outputs) como los impactos (outcomes) que generan son observables. En este caso, la medición se simplifica y el hacer pública dicha información tiene una mayor probabilidad de generar valoraciones adecuadas del desempeño de la organización. En segundo lugar están las organizaciones que denomina procedimentales, que son aquellas en que es posible observar los productos de su acción pero no los impactos que generan. En este caso, el vínculo entre los resultados inmediatos de las actividades y la transformación de las situaciones económicas, políticas, sociales y/o culturales consideradas indeseables está sujeto a debate y a polémica. Si bien la organización está en posibilidad de transparentar por medio de mediciones lo que produce, el juicio sobre si estos productos (bienes o servicios) son de valor público resulta complejo. En tercer lugar están las organizaciones que Wilson llama artesanales, que son aquellas en que es difícil monitorear en el día a día, por el alto nivel de complejidad de sus actividades, pero cuyos impactos son apreciables. Ejemplos de esto se tienen en el caso de las operaciones de guerra, las labores de policía y los servicios de salud. En este caso, existe una tensión entre la transparencia y el desempeño profesional, ya que la valoración del rendimiento requiere de un grado importante de especialización, por lo que normalmente es realizada por cuerpos especializados, es decir, entre pares (Radin 2006). Finalmente, Wilson identifica un cuarto tipo: el de las organizaciones que salen del paso o se las arreglan, ya que tanto los productos como los impactos son difíciles de apreciar y, por lo tanto, de medir. En estos casos transparentar el desempeño puede no sólo ser contrario a los objetivos de la organización (como es el caso de la diplomacia), sino que puede resultar impráctico, ya que no es posible contar con información confiable, sistemática y estandarizada que permita informar con claridad al público ciudadano sobre las actividades y los logros alcanzados.

Como podemos observar, la transparencia y la gestión del desempeño pueden reforzarse mutuamente en el caso de las organizaciones de producción. Sin embargo, en los otros tres casos la relación es más complicada. Es, por lo tanto, esperable que aquellas organizaciones que tienen dificultades para medir tanto sus productos como sus impactos sean también las que más reticencia muestren a abrir su información, pues se encuentran en una situación de mayor vulnerabilidad a la manipulación política o interesada de dichos datos. 


\section{TransparenCIA Y SUS EFECTOS SOBRE EL COMPORTAMIENTO}

Con respecto al comportamiento de los funcionarios, ya se señaló que la transparencia puede generar algunos comportamientos adecuados, es decir que generen aún más transparencia y/o mejoren el rendimiento de programas y servicios. Sin embargo, también puede generar patrones de actuación indeseables, es decir, comportamientos que no conducen a una mejora del rendimiento en la práctica y/o que erosionan la confiabilidad de la información, por lo que en realidad se reduce efectivamente la calidad de la información disponible. Moynihan (2010) identifica cuatro posibles reacciones frente a las iniciativas para transparentar el desempeño organizativo, a saber:

1. Pasiva. Cuando se realiza el menor esfuerzo posible para cumplir con los requerimientos de generación y diseminación de información. Esto resulta racional si dichos requerimientos parecen pasajeros, si los políticos y el público hacen poco caso de esa información y/o si el liderazgo de la organización no encuentra algún beneficio ligado a dichos requerimientos. En este caso, la respuesta de tipo ritual ante estos requerimientos de transparencia no necesariamente genera mejor información para el público ni mayor desempeńo organizativo.

2. Política. Utilizar los datos para definir el propósito de la organización y movilizar apoyo en la arena política. Así, la transparencia permite a la propia organización mejorar su posicionamiento en el entorno, por lo que aumenta sus probabilidades de hacerse de recursos requeridos para mejorar su rendimiento.

3. Perversa. Ante un grado intenso de presión, la organización cumple con los requerimientos pero a costa de los objetivos implícitos o no medidos del proyecto, plan o programa. Esta respuesta representa en caso más extremo de contradicción entre transparencia y desempeño, donde la primera afecta al segundo.

4. Propositiva. Los funcionarios hacen uso de la información del desempeño para mejorar la gestión de su programa u organización. Esto puede suceder a partir de un aprendizaje derivado de la comparación entre las metas y lo alcanzado, o al ligar la información del desempeño a ciertas sanciones o premios. Al contrario de la situación antes descrita, en este caso, tanto transparencia como desempeño pueden reforzarse mutuamente.

De esta forma, dependiendo del tipo de actitudes que asuman los funcionarios frente a la mancuerna de transparencia y gestión del 
desempeño, se tendrán resultados positivos o contradictorios. De particular interés ha sido el estudio de expresiones concretas de las respuestas pasiva y perversa (De Brujin 2002, Radin 2006, Talbot 2010). Algunas de ellas son las siguientes:

1. Enfoque estrecho en lo que es medido. En algunos casos, aquello medido y valorado como buen desempeño se puede lograr a costa de otros aspectos importantes del programa o servicio que no están incluidos por el sistema de gestión del desempeño. Esto implica que aquello que se mide y se transparente puede, paradójicamente, obscurecer otros aspectos del servicio, erosionando su calidad vista de manera comprehensiva ${ }^{6}$.

2. Sustitución de esfuerzo. Al clarificar los aspectos que serán medidos y puestos a disposición del público, los funcionarios desplazan su atención, recursos y tiempo hacia las tareas directamente relacionadas con lo que se medirá, dejando de lado otras que pueden ser importantes (Kelman y Friedman 2009). Ejemplo de esto es concentrarse en los alumnos que están cerca de lograr los niveles de competencia exigidos, dejando de lado la atención a aquellos más rezagados y/o a los más aventajados. En este caso, la transparencia también erosiona el desempeńo, en la medida que se ocultan los comportamientos indeseados.

3. Aparición de juegos estratégicos. En este caso, el problema es que la combinación de transparencia y gestión del desempeño puede no eliminar por completo las asimetrías de información que existe entre los funcionarios y el público en general (o sus representantes). Aquellos que producen el servicio tienen más información que quienes evalúan sus resultados y aplican sanciones. La consecuencia es que existe la posibilidad de engañar mediante el establecimiento de objetivos que son fáciles de lograr o la difusión de información inexacta sobre los resultados realmente obtenidos, entre otros (Kelman y Friedman 2009). Aunque éste es un riesgo, no debe exagerarse sus efectos, ya que no existe evidencia contundente de que sean fenómenos generalizados. Por ejemplo, Talbot (2010) calcula que en el caso de las listas de espera por operaciones no urgentes en el Reino Unido, el ocultamiento de pacientes es de aproximadamente $5 \%$.

Los comportamientos expuestos tienen como resultado que la información transparentada no sea confiable para emitir un juicio sobre el

6 Medir tantos aspectos del servicio como sea posible es una manera de hacer frente a este problema. Sin embargo, entre más complejo sea el sistema más difícil es interpretar la información que genera (Broadbent 2003). 
desempeńo real de las organizaciones y/o empeoran el rendimiento de dichas organizaciones. Si bien se cuenta con un número creciente de estudios que permiten establecer que las actitudes de tipo perverso no necesariamente son generalizadas, es esperable que en contextos de escasez de recursos o de alta polarización del debate público se vuelvan más recurrentes como medio de defensa ante posibles recortes de personal o presupuestales.

\section{TransPaRenCia Y PROFESIONALISMO}

Gran parte de las reformas de la administración pública de los años recientes han tenido como común denominador la desconfianza frente al aparato burocrático y los cuerpos profesionales ligados a las funciones del estado social de derecho: médicos, maestros, científicos, trabajadores sociales, entre otros. Frente a tareas de alta complejidad que requieren grados importantes de especialización y capacidad de respuesta ante las características de cada caso particular que se atiende, la administración pública contemporánea ha adoptado la forma de burocracias profesionales como las define Mintzberg:

[L]a burocracia profesional confía para la coordinación y su parámetro de diseño asociado [es la] capacitación y enseñanza. Contrata especialistas debidamente capacitados y enseńados profesionales- [...] y luego les da considerable control sobre su propio trabajo (Mintzberg 1989: 159).

Esta receta requiere de un alto grado de confianza en la labor de dichos expertos, aunque a posteriori pueda valorarse su desempeño (Romzeck y Dubnick, citados en Radin 2006: 55-56). Esta rendición de cuentas por lo regular toma la forma de una revisión por parte de otros profesionales del mismo campo de actividad, es decir, realizada por pares. Day y Klein (1987) denominan a esto rendición de cuentas profesional. En este sentido, una profesión se entiende como una comunidad de personas especializadas en un campo de actividad social que comparten o se someten a definiciones compartidas respecto a qué se considera un comportamiento adecuado.

Analistas críticos han señalado que el creciente poder de las profesiones dentro del aparato gubernamental las ha hecho inmunes antelos mecanismos de control democrático. De allí que se hayan hecho esfuerzos por acotar su autonomía. En buena medida, la gestión del desempeño aspira a ofrecer a los ciudadanos y sus representantes un medio para valorar el trabajo de los expertos, por medio de mediciones que se centran en disminuir las asimetrías de información que abren la puerta a que intereses particulares distorsionen el interés general (Clarke 2005). Así, la transparencia respecto del desempeño organizativo se vuelve una herramienta para dotar de poder 
de control a la ciudadanía en general, devolviendo el carácter democrático a las actuaciones de los cuerpos profesionales.

Arellano (2005), Radin (2006) y Talbot (2010) han señalado que esta lógica parte de un paradigma basado en el modelo del homo economicus, orientado a maximizar su interés que responde a los incentivos positivos y negativos, premios y sanciones. Sin embargo, dicha perspectiva no considera la importancia de los lazos de confianza dentro de las instituciones que son un elemento fundamental no sólo de identidad organizativa sino también fuente de compromiso con valores públicos y con la mejora permanente de las actuaciones. En este sentido, mayor transparencia basada en un monitoreo del desempeño con apego a indicadores y modelos estandarizados puede resultar tanto en una disminución de la calidad de atención a los casos que se apartan de la norma, como enviar una señal de que no existe confianza en el personal, lo que puede generar la erosión de la motivación del personal, con sus consecuentes efectos negativos sobre la productividad y permanencia.

\section{Transparencia EX ANTE Y EX POST}

Finalmente, la variable tiempo resulta muy importante para poder estimar los efectos que tiene la transparencia sobre el comportamiento y desempeño organizativos. Una de las premisas centrales de la gestión del desempeño es que se debe pasar de las formas tradicionales de control burocrático por medio de normas, regulaciones y supervisión, hacia modelos de coordinación del trabajo con base en la rendición de cuentas ex post, es decir, por resultados, que ofrezcan grados de libertad o autonomía a los gerentes públicos para aprovechar sus conocimientos, habilidades y experiencia (Peters 2005). Sin embargo, las demandas por transparentar el desempeńo pueden ser vividas por los funcionarios como una nueva forma de control, lo que puede generar comportamientos recelosos, temerosos y adversos al riesgo. Es por esta razón que es importante entender en qué momento se hace público el desempeño, pues esto puede afectar de formas distintas el comportamiento de la organización. Siguiendo el ciclo de gestión de las políticas y programas, se puede transparentar el desempeño organizativo en diferentes momentos o etapas del mismo:

1. Ex ante. Se refiere a hacer público el nivel de desempeño que se desea alcanzar, por ejemplo, el ańo siguiente. Normalmente, cuando se transparentan los objetivos es posible hacer comparaciones con, por citar un caso, las plataformas político-electorales defendidas por el partido ganador de unas elecciones, de manera que se pueda valorar nivel el cumplimiento del gobierno respecto de sus promesas de campaña. Sin embargo, este tipo de transparencia puede generar el 
establecimiento de objetivos y metas fáciles de alcanzar que resultan, por lo tanto, poco útiles tanto para mejorar el desempeńo como para entender lo que realmente se propone hacer el gobierno.

2. Ex dure. Se refiere a hacer pública la información que permite saber lo que está ocurriendo durante la implementación de una política o programa. Este es el caso que mayores constreñimientos supone para el funcionariado, ya que la transparencia se vive como una exigencia de ofrecer información en tiempo real (Held 2006), sin que exista un espacio de tiempo dedicado exclusivamente a la ejecución de acciones. También resulta el tipo de transparencia más caro, por las cargas administrativas que implica la respuesta constante a las exigencias de información (Van Thiel y Leeuw, citados en Radin 2006).

3. Ex post. Se refiere a la comparación entre los objetivos, los resultados planeados y los resultados obtenidos. También llamada transparencia retrospectiva, es el nivel mínimo de transparencia que debe esperarse y el que también permite un mayor margen de maniobra a los funcionarios. En este sentido, la organización vive momentos de generación de información y rendición de cuentas que, una vez concluidos, dan paso a un periodo en que es posible concentrarse en realizar las actividades y los objetivos (Held 2006).

4. De mejora. Se refiere a transparentar los compromisos de mejora del desempeño futuro de la organización que resultan del análisis o revisión del rendimiento pasado. Es decir, se refiere a hacer públicos los objetivos de mejora del propio funcionamiento de la administración en los siguientes meses o años. Este tipo de transparencia podría fomentar una actitud propositiva, de aprendizaje permanente, que podría ser la más productiva en términos de aprovechamiento de la información de desempeño generada a lo largo del ciclo de la política (De Brujin 2002, Metzenbaum 2006).

Es de esperarse, por lo tanto, que entre mayores exigencias de transparencia se hagan a lo largo del proceso de la política, menor sea el grado de autonomía que se otorga a los gerentes para enfocarse en lograr resultados, por lo que tenderán a comportarse de manera recelosa y defensiva. De allí la importancia de diseñar con cuidado los regímenes de desempeńo, de manera que el momento en que la información sobre el rendimiento organizativo se hace pública aumente las posibilidades de generar esfuerzos por lograr mejores resultados. Es decir, se debe evitar que la transparencia se vuelva una fuente más de inflexibilidad del aparato burocrático. 


\section{CONCLUSIÓN}

Es común que las personas e instituciones que han apoyado la diseminación de las ideas y técnicas asociadas a la NGP en todo el mundo tiendan a acentuar las posibles relaciones de reforzamiento mutuo que éstas tienen con el ideal de la transparencia gubernamental. En muchas instancias, sin duda, estas relaciones positivas efectivamente ocurren, generando círculos virtuosos entre el gerencialismo, el desempeño gubernamental y el acceso a más y mejor información sobre la actividad gubernamental. En México, por ejemplo, nunca antes se había tenido tanta información sobre el desempeño de los programas del sector social, gracias a la publicación de ejercicios recurrentes de medición del desempeño y la evaluación de los mismos (Caso Raphael 2010).

Sin embargo, como se ha visto a lo largo de este trabajo, lograr el equilibrio adecuado entre gestión del desempeño y transparencia es un reto difícil de resolver en la práctica. Por un lado, la falta de transparencia puede generar espacios de ineficiencia o de decisiones que favorecen intereses particulares. Una exigencia de transparencia permanente, por el otro, genera costos de cumplimiento e incentiva comportamientos acomodaticios, tímidos y adversos a tomar riesgos, lo que puede resultar en una merma a la calidad de la información que se ofrece y/o una erosión del propio rendimiento gubernamental. Las relaciones entre gestión del desempeño y transparencia son más complejas de lo que a primera vista aparentan.

De lo anterior se desprende que es riesgoso tomar modelos o herramientas de gestión del desempeño de manera estandarizada, sin adaptarlas a los contextos y necesidades específicos de aquello que se quiere medir o transparentar. En dicha adecuación es necesario considerar el tipo de actividades que se desea mejorar, valorar los costes financieros y en términos de distorsión de los comportamientos que pudieran derivarse de las reformas propuestas y asegurar que los mecanismos de medición y transparencia son oportunos y relevantes. En este sentido, se requiere lograr un equilibro entre las expectativas de información que tiene la sociedad respecto a ciertos ámbitos de actuación del gobierno y el ámbito de actuación profesional que se quiere dar a los gerentes públicos para que alcancen ciertos resultados. Por desgracia, en ciertas circunstancias una mayor cantidad de información no necesariamente conduce o es compatible con un mayor rendimiento, así como en ocasiones el tener mayor información no necesariamente permite hacer una valoración adecuada del desempeño alcanzado. 


\section{REFERENCIAS}

Aguilar Villanueva, L. (2006). Gobernanza y Gestión Pública. México: Fondo de Cultura Económica.

(2011). Gobernanza Pública para Obtener Resultados: Marco Conceptual y Operacional. Nueva York: Naciones Unidas.

Arellano, D. (2005). La Transparencia desde la Teoría de las Organizaciones. En Merino, M. (coord.), Transparencia. Libros, Autores e Ideas. México: CIDE-IFAI.

Aucoin, P. y Heintzman, R. (2000). The Dialectics of Accountability for Performance in the Public Management Reform. International Review of Administrative Sciences, 66 (1), 45-55.

Broadbent, J. (2003). Comprehensive Performance Assessment: The Crock of Gold at the End of the Rainbow? Public Money and Management, 23 (1), 712-725.

Caso Raphael, A. (2010). La Gestión Pública por Resultados y su Impacto en la Política Presupuestal de México. Ponencia presentada en el Seminario Internacional de Evaluación de Políticas Públicas, 17 de noviembre de 2010, México.

Chun, Y. H. y Rainey, H. G. (2006). Consequences of Goal Ambiguity in Public Organizations. En Boyne, G. et al. (Eds.), Public Service Performance: Perspectives on Measurement and Management. Cambridge: Cambridge University Press.

Clarke, J. (2005). Performing for the Public: Doubt, Desire, and the Evaluation of Public Services. En Gay P. D. (Ed.), The Values of Bureaucracy. Oxford: Oxford University Press.

Comisión de Auditoría. (2000). Aiming to Improve: The Principles of Performance Measurement, Londres: Audit Commission.

Day, P. y Klein, R. (1987). Accountabilities: Five Public Services. Londres: Tavistock.

De Brujin, H. (2001). Managing Performance in the Public Sector. Londres: Routledge.

Dirección de Presupuestos (DIPRES). (2003). Programa de Mejoramiento de la Gestión Año 2004. Santiago de Chile: Dirección de Presupuestos. 
Fox, J. (2007). The Uncertain Relationship between Transparency and Accountability. Development in Practice, 17 (4-5), 663-671.

Guerrero, J.(2005). Transparencia: De la Abstracción a la Operación de un Concepto. En Merino, M. (coord.), Transparencia. Libros, Autores e Ideas. México: CIDE-IFAI.

Heinrich, C. (2002). Outcome-Based Performance Management in the Public Sector: Implications for the Government Accountability and Effectiveness. Public Administration Review, 62 (6), 712-725.

Heald, D. (2006). Varieties of Transparency. En Hood, C. y Heald, D. (Eds.), Transparency: The Key to Better Governance? Proceedings of the British Academy (135). Oxford: Oxford University Press.

Hood, C. (1991). A Public Management for All Seasons? Public Administration Review, 68 (1), 2-19.

(2006). Transparency in Historical Perspective. En Hood, C. y Heald, D. (Eds.), Transparency: The Key to Better Governance? Proceedings of the British Academy (135). Oxford: Oxford University Press.

James, O. (2005), Performance Targets for Executive Agencies and Public Sector Externalities in the UK. En Drewry, G., Greve, C. y Tanquerel, T. (Eds.), Contracts, Performance Measurements, and Accountability in the Public Sector. Amsterdam: IOS Press.

Kelman, S. y Friedman, J. (2009). Performance Improvement and Performance Dysfunction: An Empirical Examination of Distortionary Impacts of the Emergency Room Wait-Time Target in the English National Health Service. Journal of Public Administration Research and Theory, 19 (4), 917-946.

Kondo, S. (2002). Fostering Dialogue to Strengthen Good Governance. En OECD, Public Sector Transparency and Accountability Making it Happen. Paris: OECD.

Metzenbaum, S. (2009). Rendición de Cuentas del Desempeño: Los Cinco Pilares y las Seis Prácticas Esenciales. En Pardo, M. y Velasco, E. (Coords.), La Gerencia Pública en América del Norte. Tendencias Actuales de la Reforma Administrativa en Canadá, Estados Unidos y México. México: El Colegio de México.

Mintzberg, H. (1989). Diseño de Organizaciones Eficientes. Buenos Aires: El Ateneo. 
Moynihan, D. (2010). From Performance Management to Democratic Performance Governance. En O'Leary, R., Van Slyke, D. y Kim, S. (Eds.), The Future of Public Administration Around the World. The Minnowbrook Perspective. Washington: Georgetown University Press.

Peters, G. (2005). Squaring Several Circles: Coordination, Performance and Accountability. En Drewry, G., Greve, C. y Tanquerel, T. (Eds.), Contracts, Performance Measurements, and Accountability in the Public Sector. Amsterdam: IOS Press.

Radin, B. (2006). Challenging the Performance Movement. Accountability, Complexity and Democratic Values. Washington: Georgetown University Press.

Stewart, J. (1984). The Role of Information in Public Accountability. En Hopwood, A. y Tomkins, C. (Eds.), Issues in Public Sector Accounting. Oxford, Phillip Allan.

Talbot, C. (2010). Theories of Performance. Organizational and Service Improvement in the Public Sector. Oxford: Oxford University Press.

Van Dooren, W, Bouckaert, G y Halligan, J. (2010). Performance Management in the Public Sector. Oxford: Routledge.

Vergara, R. (2008). La Transparencia como Problema. México: IFAI.

Wilson, J. Q. (1989). Bureaucracy. What Government Agencies Do And Why They Do It. Nueva York: Basic Books.

Wignaraja, K. y Gebrehiwot, S. (2011). Freedom of Information in the Fight against Poverty and Promotion of Freedoms. Disponible en http://pressroom.ipc-undp.org/op-ed-freedom-of-information-in-thefight-against-poverty-and-promotion-of-freedoms/ [02-10-2013].

Recibido: 31-08-2013

Aceptación de la versión final: 11-12-2013 